University of Wollongong

Research Online

Faculty of Social Sciences - Papers (Archive) Faculty of Arts, Social Sciences \& Humanities

$1-1-2020$

Better Health Choices: Feasability and preliminary effectiveness of a peer delivered healthy lifestyle intervention in a community mental health setting

Peter James Kelly

University of Wollongong, pkelly@uow.edu.au

Amanda Baker

University of Wollongong, abaker@uow.edu.au

Naomi Fagan

University of Wollongong

Alyna Turner

Frank P. Deane

University of Wollongong, fdeane@uow.edu.au

See next page for additional authors

Follow this and additional works at: https://ro.uow.edu.au/sspapers

Part of the Education Commons, and the Social and Behavioral Sciences Commons

Research Online is the open access institutional repository for the University of Wollongong. For further information contact the UOW Library: research-pubs@uow.edu.au 


\title{
Better Health Choices: Feasability and preliminary effectiveness of a peer delivered healthy lifestyle intervention in a community mental health setting
}

\author{
Abstract \\ 2019 Elsevier Ltd Background: To reduce smoking and improve other health behaviours of people living \\ with severe mental illness, healthy lifestyle interventions have been recommended. One approach to \\ improving the availability of these types of interventions is to utilise the mental health peer workforce. \\ The current study aimed to evaluate the feasibility of peer-workers facilitating a telephone delivered \\ healthy lifestyle intervention within community based mental health settings. The study also examined \\ preliminary outcomes of the intervention. Methods: The study was conducted as a randomised controlled \\ feasibility trial. In addition to treatment as usual, participants randomised to the Treatment Condition \\ were offered BHC. This was an 8-session telephone delivered coaching intervention that encouraged \\ participants to decrease their smoking, increase their intake of fruit and vegetables, and reduce their \\ leisure screen time. Participants in the waitlist Control Condition continued to complete treatment as \\ usual. All participants were engaged with Neami National, an Australian community mental health \\ organisation. Peer-workers were also current employees of Neami National. Results: Forty-three \\ participants were recruited. The average number of sessions completed by participants in the Treatment \\ Condition was 5.7 (SD = 2.6; out of 8-sessions). Seventeen participants $(77 \%)$ completed at least half of \\ the sessions, and nine participants (40\%) completed all eight sessions. Participant satisfaction was high, \\ with all participants followed up rating the quality of the service they received as 'good' or 'excellent'. \\ When compared to the Control Condition, people in the Treatment Condition demonstrated greater \\ treatment effects on smoking and leisure screen time. There was only a negligible effect on servings of \\ fruit and vegetable. Conclusions: Results were promising regarding the feasibility of peer-workers \\ delivering BHC. Good retention rates and high consumer satisfaction ratings in the Treatment Condition \\ demonstrated that peer-workers were capable of delivering the intervention to the extent that consumers \\ found it beneficial. The current results suggest that a sufficiently powered, peer delivered randomised \\ controlled trial of $\mathrm{BHC}$ is warranted. Study registration: Australian New Zealand Clinical Trials Registry \\ (ANZCTR; Trial ID ACTRN123615000564550). \\ Disciplines \\ Education | Social and Behavioral Sciences

\section{Publication Details} \\ Kelly, P., Baker, A., Fagan, N., Turner, A., Deane, F., McKetin, R., Callister, R., Collins, C., Ingram, I., \\ Wolstencroft, K., Townsend, C., Osborne, B. \& Zimmermann, A. (2020). Better Health Choices: Feasability \\ and preliminary effectiveness of a peer delivered healthy lifestyle intervention in a community mental \\ health setting. Addictive Behaviors, 103
}

\section{Authors}

Peter James Kelly, Amanda Baker, Naomi Fagan, Alyna Turner, Frank P. Deane, Rebecca McKetin, Robin Callister, Clare Collins, Isabella Ingram, Keren Wolstencroft, Camilla Townsend, Briony Osborne, and Adam Zimmermann 
PEER-DELIVERED Better Health Choices

Better Health Choices: feasability and preliminary effectiveness of a peer delivered healthy lifestyle interventon in a community mental health setting.

Peter J. Kelly ${ }^{1}$, Amanda L. Baker ${ }^{2}$, Naomi L. Fagan ${ }^{1}$, Alyna Turner ${ }^{2}$, Frank Deane ${ }^{1}$, Rebecca McKetin $^{3}$, Robin Callister ${ }^{2}$, Clare Collins ${ }^{2}$, Isabella Ingram ${ }^{1}$, Keren Wolstencroft ${ }^{4}$, Camilla Townsend ${ }^{1}$, Briony A. Osborne ${ }^{1}$, and Adam Zimmermann ${ }^{4}$

${ }^{1}$ School of Psychology and Illawarra Health and Medical Research Institute, University of Wollongong, Wollongong, Australia

${ }^{2}$ University of Newcastle, Newcastle, Australia

${ }^{3}$ National Drug Research institute, Curtin University, Perth, Australia

${ }^{4}$ Neami National, Victoria, Australia.

Author for correspondence: Peter J. Kelly, School of Psychology, University of Wollongong, Wollongong, NSW, Australia, 2500. Telephone: 024239 2382, Email: pkelly@uow.edu.au

Keywords: peer, lived experience, healthy lifestyle, smoking, sedentary, schizophrenia, severe mental illness 


\section{PEER-DELIVERED Better Health Choices}

Better Health Choices: feasability and preliminary effectiveness of a peer delivered healthy lifestyle interventon in a community mental health setting. 


\begin{abstract}
Background: To reduce smoking and improve other health behaviours of people living with severe mental illness, healthy lifestyle interventions have been recommended. One approach to improving the availability of these types of interventions is to utilise the mental health peer workforce. The current study aimed to evaluate the feasibility of peer-workers facilitating a telephone delivered healthy lifestyle intervention within community based mental health settings. The study also examined preliminary outcomes of the intervention.
\end{abstract}

Methods: The study was conducted as a randomised controlled feasibility trial. In addition to treatment as usual, participants randomised to the Treatment Condition were offered BHC. This was an 8-session telephone delivered coaching intervention that encouraged participants to decrease their smoking, increase their intake of fruit and vegetables, and reduce their leisure screen time. Participants in the waitlist Control Condition continued to complete treatment as usual. All participants were engaged with Neami National, an Australian community mental health organisation. Peer-workers were also current employees of Neami National.

Results: Forty-three participants were recruited. The average number of sessions completed by participants in the Treatment Condition was 5.7 ( $S D=2.6$; out of 8 -sessions). Seventeen participants $(77 \%)$ completed at least half of the sessions, and nine participants (40\%) completed all eight sessions. Participant satisfaction was high, with all participants followed up rating the quality of the service they received as 'good' or 'excellent'. When compared to the Control Condition, people in the Treatment Condition demonstrated greater treatment effects on smoking and leisure screen time. There was only a negligible effect on servings of fruit and vegetable. 
PEER-DELIVERED Better Health Choices

Conclusions: Results were promising regarding the feasibility of peer-workers delivering BHC. Good retention rates and high consumer satisfaction ratings in the Treatment Condition demonstrated that peer-workers were capable of delivering the intervention to the extent that consumers found it beneficial. The current results suggest that a sufficiently powered, peer delivered randomised controlled trial of BHC is warranted.

Study registration: Australian New Zealand Clinical Trials Registry (ANZCTR; Trial ID ACTRN123615000564550). 
Better Health Choices: feasability and preliminary effectiveness of a peer delivered healthy lifestyle interventon in a community mental health setting.

\section{Introduction}

It is well established that people living with severe mental illness demonstrate a high rate of lifestyle diseases (i.e. cardiovascular disease, cancer, diabetes) ${ }^{1}$. Engagement in a range of unhealthy lifestyle behaviours makes people living with severe mental illness more susceptible to developing these diseases. This includes high rates of smoking ${ }^{2}$ and alcohol use disorder $^{3}$, high rates of sedentary behaviour and physical inactivity ${ }^{4,5}$, and poor dietary behaviours including low intake of fruit and vegetables ${ }^{1,6}$. Guidelines for addressing the prevention of lifestyle diseases in people living with severe mental illness recommend that health risk behaviours be addressed (i.e. smoking, poor diet, physical inactivity, sedentary behaviour and alcohol misuse) $)^{5,7}$. People living with severe mental illness experience a range of unique barriers that prevent the development and maintenance of healthy lifestyle behaviours, including psychiatric symptoms, and medication side effects. As such, there is a need for multiple health risk behaviour change interventions that are accessible and tailored for people living with severe mental illness.

Better Health Choices (BHC) is an 8-session telephone-delivered healthy lifestyle intervention that was developed for people living with severe mental illness. It encourages participants to decrease their smoking and alcohol use, improve their diet by increasing their intake of fruit and vegetables, and reduce their leisure screen time. The development of BHC has been previously described ${ }^{8}$. The focus on fruit and vegetable intake and sedentary behaviour was largely influenced by the work of Spring and colleagues ${ }^{9}$. In this study, people from the general population with four cardiometabolic risk behaviours (i.e. low fruit and vegetable intake, high fat diets, low physical activity, and high levels of leisure screen time) were randomised to one of four 3-week interventions that focused on one dietary and 
one activity goal each. The most effective intervention aimed to increase fruit and vegetable intake and decrease leisure screen time. This combination was associated with significant, large and sustained improvements in fruit and vegetable intake, screen time, and serendipitously, saturated fat intake. Traditional 'dieting' (decreasing saturated fat \& increasing physical activity) achieved lower levels of improvement than did the other three treatments $(\mathrm{p}<.001)$. A pilot of BHC has previously been conducted ${ }^{8}$. Participant retention in this pilot study was good, with 19 (95\%) participants completing the intervention, and 17 (85\%) participants completing follow up assessment. Preliminary outcomes were promising, with statistically significant improvements in fruit consumption, overall diet quality, leisure screen time, overall sitting time, and global functioning. There were also improvement trends in vegetable consumption, quality of life, time spent walking, and reduction in smoking (for participants who smoked tobacco at pre-treatment). A limitation of this pilot study was that it was conducted under circumstances that do not necessarily reflect routine care. Psychologists or clinical psychologists delivered the program (including PK, AB, AT) and participants were higher functioning than the general population of people living with schizophrenia ${ }^{10}$. The next step in the development of BHC was to examine feasibility when delivered as part of a community based mental health service.

A recent systematic review, focused on preventative health interventions delivered by peer workers, highlighted that there was potential for peer-workers to play an important role in this area ${ }^{11}$. Studies included in this review included interventions that were either peer-led or co-facilitated by peer specialists (i.e. "people with a lived experience recovering from a mental illness") and other health professionals (p. 85). The majority of the studies examined manualized interventions, and were either delivered in group formats, individual formats, or combined formats. The review did not specifically address if any of the interventions were primarily delivered using the telephone. The review concluded that there was 'limited' 
current evidence for interventions targeting smoking or physical activity and 'beneficial' support for diet ${ }^{11}$. For example, the review identified five studies that reported smoking outcomes. Two of the studies demonstrated statistically significant reductions in smoking, with the remaining three studies reporting reductions in smoking that were either not statistically significant or the authors did not report significance. The review did not identify any interventions that specifically targeted alcohol, however, there is evidence for the positive role of peer delivered support services for substance use disorders ${ }^{12}$. Whilst there is potentially a strong role for peer-workers to play in supporting the delivery of healthy lifestyle interventions, there remains a need to establish feasible and effective interventions that can be used as part of routine care. One approach to improving the evidence in this field is to consider adapting established manualized interventions, that draw on evidence based behavioural approaches, for delivery by peer-workers.

The primary aim of the current study was to evaluate the feasibility of delivering BHC in circumstances that reflect routine care, namely peer worker delivery of BHC to consumers of a community mental health service. Feasibility was assessed in terms of Treatment and Control Condition retention, participant satisfaction with BHC, and the ability for the peerworkers to demonstrate the use of behavioural counselling skills. Preliminary outcomes of the program were also examined. This included the 4 primary behaviours targeted as part of BHC: smoking, leisure screen time, diet (i.e. fruit and vegetable intake), and alcohol intake. Physical activity, overall diet quality and well-being (i.e. psychological distress and quality of life) were also examined to see if there were any serendipitous effects of the intervention on these variables.

\section{Materials and Methods}

\section{Design and setting.}


The study was conducted as a randomised controlled feasibility trial. Participants were randomly assigned to (1) Treatment Condition (BHC) or (2) Control Condition. All participant involvement in the study occurred over the phone, including contact with the assessment officers and peer-workers. The protocol was developed according to 2013 Standard Protocol Items Recommendations for Intervention Trials (SPIRIT) guidelines ${ }^{13}$, was registered with the Australian New Zealand Clinical Trials Registry (Trial ID ACTRN123615000564550), and is reported using the CONSORT 2010 checklist for pilot/feasibility trials ${ }^{14}$. The study was funded by the Research Trust Fund of Schizophrenia Fellowship of NSW and the University of Wollongong's Human Research Ethics Committee provided approval to conduct the study (HE14/345).

\section{Participants.}

Consumers. Participants were recruited from Neami National, an Australian community mental health organisation whose mission is "to improve mental health and wellbeing in local communities" ${ }^{15, p .2}$. Inclusion criteria were: current Neami client, being $\geq 18$, and identification of a health-related goal. Exclusion criteria were being enrolled in a Neami face-to-face healthy lifestyle peer support intervention, being currently enrolled in a Neami National homelessness service, hearing impairment precluding telephone interview, acute suicidality, pregnancy, or acquired brain injury.

Peer-workers. The seven peer-workers were drawn from the Neami National workforce where they were all employed as peer workers. Consistent with previous definitions used in the field ${ }^{11}$, peer workers referred to people with their own lived experience of mental illness ${ }^{3}$. Neami National provides peer workers with training in the Collaborative Recovery Model $^{16}$, suicide intervention and prevention, coaching and motivational interviewing, group facilitation, and training on using lived experience appropriately and effectively. Peer workers in the study were paid for their involvement. 
Sample size. Being a feasibility trial, the study was not powered to detect a significant treatment effect.

Randomisation. Randomisation was managed independently by a member of the research team $(\mathrm{RM})$, using a computer-generated randomisation schedule. Four groups of sequenced randomisation envelopes were provided, grouped by gender and presence or absence of a diagnosed psychotic disorder. When baseline assessment was completed, the research assistant would select an envelope from the appropriate group, and would open it to find which condition the participant was allocated to.

\section{Procedure.}

Participant recruitment procedure. A project officer at Neami National (who was also a peer worker) liaised with Neami National services, encouraging case workers to discuss the project with consumers. If consumers were interested, participants would fill in an expression of interest form which would be sent to the research team for further follow-up. Participants were paid \$20 (in vouchers) for each assessment that they completed throughout the study.

Peer-worker training and support. The training was facilitated by the research team over two days, in a face-to-face group setting. Peer-workers then participated in a simulated first session, which was audio-recorded, and reviewed during supervision to ensure that peer workers reached proficiency. Telephone supervision with peer-workers occurred on a weekly-to-fortnightly basis. Peer-workers had the opportunity to contact supervisors with additional concerns or queries, to discuss urgent action plans, and for debriefing. Peerworkers also had access to Neami's on-call support system and Employee Access Program.

Control Condition. Participants in the Control Condition received standard guidebooks and pamphlets provided by SANE Australia outlining information about 
cardiometabolic risk factors. For the duration of the intervention and follow up period (16 weeks total), participants in the control condition continued with treatment as usual, which, whilst variable between participants, would typically include engagement with a psychiatrist and a Neami support worker. In line with ethics procedures, Control Condition participants were offered the intervention following completion of follow-up assessments.

Treatment Condition. In addition to treatment as usual, participants in the Treatment Condition were offered the opportunity to complete BHC. As previously described by Baker

${ }^{8}$, BHC is an eight session manualised telephone-delivered intervention that uses motivational interviewing and cognitive behavioural strategies to target: (1) low fruit and vegetable intake. Consistent with Baker et al. ${ }^{8}$, a box of fruit and vegetables were delivered between sessions 1 and 2 to assist initiation of behaviour change. (2) Leisure screen time (i.e. watching TV, using computers/tablets at home). Conversations with participants focused on maximising leisure screen time for perceived meaningful activities (e.g. shared activities with friends) and reducing leisure screen time that was not meaningful for the participant (e.g. just watching anything on television to relieve boredom). Where appropriate, (3) smoking and (4) alcohol were also targets. Written baseline assessment feedback was provided to the persons case manager and medical specialist (e.g. general practitioner, psychiatrist). Where clinically appropriate, the letter also highlighted the potential benefits of nicotine replacement therapy. The peer workers also reinforced the importance of nicotine replacement therapy to the participants.

\section{Measures.}

All assessments were completed by research assistants blind to treatment condition, with the exception of questions relating to satisfaction with the program. 
PEER-DELIVERED Better Health Choices

Treatment satisfaction and retention. Satisfaction with BHC was assessed using the Client Satisfaction Questionnaire (CSQ-8) ${ }^{17}$ completed at the 16-week assessment. Treatment group retention was assessed as the number of sessions completed.

Behavioural counselling techniques. Audiotapes of the counselling sessions were rated using the Behaviour Change Counselling Index BECCI; ${ }^{18}$. The overall BECCI practitioner score was calculated as the mean of all available questionnaire items and indicates how often the practitioner is engaging in behaviour change counselling skills $(0=$ Not at all, $1=$ Minimally, $2=$ To some extent, $3=\mathrm{A}$ good deal, and $4=\mathrm{A}$ great extent). As the initial two sessions of the BHC program tend to be more structured, comparison was made between BECCI scores for the first 2-sessions and the remaining sessions.

Baseline measures. Baseline assessment included all outcome measures (detailed below) in addition to demographics (e.g. age, education, employment status, marital status).

Outcome measures. Smoking was assessed using (1) seven day smoking point prevalence, and (2) questions from the tobacco section of the Opiate Treatment Index (OTI) ${ }^{19,20}$. Participants were also asked "How many cigarettes a day do you smoke?" at each of the assessment time points. Nicotine dependence was measured using the Fagerstrom Test of Nicotine Dependence ${ }^{21}$. Alcohol consumption was assessed using the Time Line Follow Back procedure for alcohol use in the preceding week ${ }^{22}$.

Fruit and vegetable intake was assessed using the fruit and vegetable subscales from the Australian Recommended Food Score (ARFS) index ${ }^{23}$. For the fruit subscale, one point is allocated for consumption of eight different fruits, including fruit salad, dried and canned fruit $\geq$ once per week and 1 -point for total fruit consumption $\geq 2 /$ day (score range $0-12$ ). For the vegetable subscale, one point is allocated for consumption of 19 different vegetables $\geq$ once per week; and one point for consuming vegetables with dinner 3-4 nights/week or two 
PEER-DELIVERED Better Health Choices

points for $\geq 5$ nights/week (score range 0-21). Servings of fruit and vegetables were also recorded.

Leisure screen time was assessed using the Marshall sedentary behavior questions targeting weekday television viewing and use of a computer or other screens at home ${ }^{24}$. The International Physical Activity Questionnaire Short Form (IPAQ-SF) was used to examine any incidental changes in physical activity. The IPAQ assesses level of activity, including walking, moderate and vigorous activity and responses were converted to metabolic equivalent task minutes per week MET $\mathrm{min} / \mathrm{wk} ;{ }^{25}$.

Psychological distress was assessed using the Patient Health Questionnaire (PHQ) ${ }^{26}$ and quality of life was assessed using the WHO-8 EUROHIS Quality of Life scale ${ }^{27}$.

Statistical methods. Analyses were run in Stata SE version 14.1 (Statacorp LP, College Station, Texas, USA). All tests were two-sided with p set at 0.05. A series of random intercept regression models were used to predict each outcome measure with the predictor being a treatment condition (Control vs. Treatment) by time (baseline vs. both follow-ups) interaction. The main effect for treatment condition reflects the difference between the treatment versus the control condition across all three time points, the time effect reflects the change at follow-up (averaged across both follow-ups) relative to baseline, and the treatment condition by time interaction reflects the effect of treatment on outcomes at follow-up relative to the control group (i.e., the treatment effect). A random intercept term was included in each model to account for repeated measures over time, implemented using Stata's xt command suite. Poisson models were used for measures of tobacco smoking, alcohol consumption, screen time and physical activity due to the nature of their distribution. All other models were linear. Sub-analysis was conducted to examine the potential effect of the intervention for people who did not already meet recommended guidelines for each of the 
health behaviours at baseline. Following the recommendations of Durlak ${ }^{28}$, adjusted Cohensd effect sizes were calculated by subtracting the baseline score from the 16-week follow-up score. This helped to account for baseline differences between the two conditions.

\section{Results}

Participant recruitment. Figure 1 shows participant numbers at each stage of the study. Between June 2015 and June 2016, 104 referrals were received by the research team, with 43 people being included in the study (see Figure 1). There was a relatively large proportion of people who returned a consent to contact form (25\%), who could not be contacted or when contacted, reported that they were not interested in participating in the study. Additionally, a further 12-people withdrew with no reason between the baseline assessment and the commencement of the intervention. The final follow-up assessment was completed on February 2017 (74\% follow-up rate at 16-weeks). Twenty-four people were allocated to the Treatment Condition and 19 to the Control Condition. See Table 1 for the participant characteristics.

Insert Table 1 and Figure 1 about here

Peer worker recruitment and retention. Seven peer workers were employed to work on the study. Following training, all seven peer workers demonstrated proficiency by completing mock sessions. The reasons the peer-workers left the study tended to be positive (e.g. securing more permanent employment, family commitments, maternity leave). However, this resulted in the large majority of the interventions being delivered by the one consistent peer worker.

Treatment satisfaction and retention. Participant satisfaction was high, with a mean treatment group CSQ-8 score of $26.9(S D=3.6)$, with all participants followed up rating the quality of the service they received as 'good' or 'excellent'. The average number of sessions 
PEER-DELIVERED Better Health Choices

completed by participants in the Treatment Condition was $5.7(S D=2.6)$ (from a total of 8 sessions).

Behavioural counselling techniques. BECCI scores $(M=1.7, S D=0.6)$ indicated that peer-workers used Behaviour Change Counselling techniques between 'minimally' and 'to some extent' across all sessions rated. Scores on the BECCI were higher in the first 2sessions $(\mathrm{M}=2.14, \mathrm{SD}=.77$, between 'to some extent' and 'a good deal'), than later sessions $(1.68, \mathrm{SD}=.60$, between 'minimally' and 'to some extent').

Tobacco smoking: At baseline, 19 participants (44\%) reported that they had smoked tobacco within the past month and were classified as 'smokers'. Of the smokers, on average they had smoked their first full cigarette at 14.9 years, started smoking daily from 17.4 years of age, reported smoking 18.1 cigarettes per day, and scored in the moderate nicotine dependence range on the Fagerstrom Test for Nicotine Dependence $(\mathrm{M}=5.0, \mathrm{SD}=2.4)$. For smokers in the Treatment Condition ( $\mathrm{n}=12$ baseline), 2 participants reported 7-day point prevalence abstinence (17\%). For smokers in the Control Condition $(n=7), 1$ person reported 7-day point prevalence abstinence at 16 weeks (14\%). There was no significant between group differences on the OTI tobacco (see Table 2 and 3). However, there was a medium to large effect demonstrated for participants who had smoked in the month prior to the baseline assessment on the OTI tobacco $(\mathrm{d}=-.8$, see Table 4$)$.

Alcohol: Rates of alcohol use were relatively low in the current study (see Table 2). On average, participants at baseline reported drinking .7 standard servings of alcohol daily. There were no significant between group differences for alcohol (see Table 3). At baseline, only 4-participants reported that they were drinking more than 2-standard drinks per day $(2=$ Treatment Condition, 2 = Control Condition). The average number of standard drinks consumed by these participants was 6.7 standard drinks per day. Unfortunately, follow-up 
PEER-DELIVERED Better Health Choices

data was not collected for the two participants in the Treatment condition. Subsequently effect sizes were not able to be calculated between the conditions.

Fruit and vegetable consumption: At baseline, participants on average consumed 1.5 servings $(\mathrm{SD}=1.2)$ of vegetables and 0.7 servings of fruit $(\mathrm{SD}=.02)$ per day. There was a small trend for improvements in amount and quality of fruit and vegetable consumption across conditions, with no significant difference between conditions. Table 4 presents the mean scores and effect sizes for those participants who did not meet the national guidelines for either fruit $(n=25)$ or vegetables $(n=41)$. There was only a negligible effect on servings per day of fruit and vegetable consumed by participants.

Leisure screen-time: At baseline, participants reported that they spent on average 267 minutes engaged in leisure screen time (i.e. television, computer) each day. There was a statistically significant treatment effect of treatment condition on leisure screen time, with participants in the Treatment Condition reporting a decrease in leisure screen time relative to the Control Condition (see Table 3). Table 4 presents the mean scores and effect sizes for those participants who reported engaging in more than 2-hours of sedentary screen time at baseline. There was a medium to large effect in favour of the Treatment Condition $(d=-.6)$.

Physical activity: At baseline, participants reported that they spent 34 minutes per week engaged in vigorous activity, 52 minutes in moderate activity, and 98 minutes walking each week. Participants in the Control Condition demonstrated greater improvements in walking and vigorous activity, whilst the Treatment Condition demonstrated greater improvements in moderate activity (see Table 3). Table 4 presents the means and effect sizes for participants who did not meet national guidelines for physical activity at baseline. There were small effects demonstrated for vigorous activity $(\mathrm{d}=.3$, in favour of Treatment Condition) and walking ( $\mathrm{d}=-.3$, in favour of Control Condition). There were very small 
PEER-DELIVERED Better Health Choices

effects for total physical activity $(\mathrm{d}=.1)$ and moderate activity (.1) in favour of the Treatment Condition.

Quality of life and symptom distress: There was a trend for quality of life and symptom distress to improve over the course of the study for both conditions. There was not a significant difference between conditions (see Table 2). There was a small effect in favour of the Treatment Condition on symptom distress $(\mathrm{d}=-.21,95 \% \mathrm{CI}=-.92, .47)$. The effect for quality of life was negligible $(\mathrm{d}=.03,95 \% \mathrm{CI}=-.66, .73)$.

Insert Table 2, 3 and 4 about here

\section{Discussion}

The current study aimed to examine the feasibility of having peer-workers deliver BHC with people living with severe mental illness. The study had good retention rates, with an average number of 6 sessions (out of 8-sessions) completed by participants. The high participant satisfaction ratings in the Treatment Condition demonstrated that peer-workers were capable of delivering the intervention to the extent that participants found it beneficial.

The current study was not powered to find statistically significant differences between conditions, meaning that all but large effect sizes would be likely to go undetected due to the high probability of two II errors. That said, promising results were seen in terms of significant reductions in tobacco smoking and leisure screen time. Although BHC does not specifically target physical activity, there were some incidental changes in activity levels that were also promising. When examining participants who did not meet recommended physical activity guidelines at baseline, there was a trend for participants in the Treatment Condition to demonstrate greater improvements than people in the Control Condition for moderate $(\mathrm{d}=$ .1) and vigorous $(\mathrm{d}=.3)$ physical activity. Surprisingly, for walking there was a small to moderate effect in favour of the Control Condition $(\mathrm{d}=.3)$. This is likely the result of the low 
MET min/wk baseline level for the Control Condition compared to the Treatment Condition (537 v 170), as both conditions demonstrated improvements in walking over the course of the study.

The increase in combined servings of fruit and vegetables for participants in the Treatment Condition ( .8 servings for total sample, 1.23 servings for participants not meeting guidelines at baseline) is consistent with what has been found in a systematic review examining increasing fruit and vegetable interventions for adults in the general population range .1 to 1.16 servings per day increase; ${ }^{29}$. Of interest, is the increase in consumption of fruit and vegetables in the Control Condition (1.2 servings for total sample, 1.5 servings for participants not meeting guidelines at baseline). It is not clear if this is the result of assessment effects, the provision of the SANE Australia reading material, or another unexplained variable. Further research should consider ways to increase vegetable intake in this population group.

The two-day training and subsequent weekly to fortnightly supervision appeared to be adequate in training the peer-workers in the BHC program. The mean BECCI ratings for peer-workers in the current study $(2.1 \pm .77$ across first 2 -sessions $)$ were consistent with those obtained by much more experienced psychologists and clinical psychologists in the previous pilot of BHC $2.4 \pm 0.3 ;{ }^{8}$. Likewise, the scores were higher than nurses with a similar level of training in behaviour change counselling during a training phase $(1.5 \pm 0.5)$ and at one year follow up $(1.6 \pm 0.7)^{30}$. However, the level of competency achieved by peer-workers in this study still indicates room for improvement. Future research may consider assessing the effectiveness of providing ongoing, specific feedback to peer-workers focused on the behavioural counselling techniques addressed as part of the BECCI.

It is important to consider the results of the current feasibility study in light of a number of limitations. The study relied on participant self-report of the health behaviours 
assessed. The study also did not require participants to meet entry criteria for all of the health behaviours being examined. This was primarily at the request of the service provider, who felt that there was an important equity issue associated with providing all clients with an opportunity to engage in a healthy lifestyle intervention. This meant that the sub-analysis should be interpreted with caution, as the cell size to calculate effect sizes were quite small. There was a relatively large proportion of participants who completed a consent to contact form who were not subsequently enrolled in the trial (25\%). Further research would benefit from examining strategies that might help to engage these people (e.g. case manager delivered services, non-telephone approaches, brief interventions). A further challenge with the current study was maintaining peer workers. As previously reported, peer workers tended to leave the project for largely positive reasons (e.g. maternity leave, securing ongoing roles, family commitments). However, it meant that there were often delays between referrals and commencement of the intervention. This likely explains the relatively high number of participants who withdrew without reason between the assessment and randomisation $(\mathrm{n}=$ 12).

The current study demonstrated that it is feasible for peer-workers to deliver a healthy lifestyle telephone intervention for people living with severe mental illness. The current results suggest that a larger randomised controlled trial is warranted. It is important that future trials are sufficiently powered to identify treatment effects for each of the health behaviours included in healthy lifestyle approaches. To ensure the ongoing and active engagement of peer-workers, future studies should prioritise the longer-term employment of peer-workers in full-time or substantial part-time paid roles. 
PEER-DELIVERED Better Health Choices

\section{Acknowledgments}

The researchers would like to thank the consumers, staff and management of Neami National who supported this study. We would also like to thank the Schizophrenia Fellowship NSW for funding the trial.

\section{Author Contribution Statement}

PK was responsible for the development and ongoing oversight of the trial, including leading the preparation of the manuscript. $\mathrm{AB}, \mathrm{AT}$ and II supported the training and supervision of the peer-workers. NF, CT, and II reviewed the audio recordings of the sessions and supported the analysis. RM developed the randomisation procedures and completed the analysis. FD, RC and $\mathrm{CC}$ provided expert advice on the intervention. $\mathrm{BO}, \mathrm{CT}, \mathrm{ND}$, and II completed baseline and follow-up assessments. KW and AZ helped to develop the project, support the peer-workers, and establish referral procedures. All authors contributed to the development of the manuscript.

\section{Conflcit of Interest Statement}

KW and AZ were employed by Neami National when the study was conducted. 


\section{References}

1.Scott D, et al.The High Prevalence of Poor Physical Health and Unhealthy Lifestyle Behaviours in Individuals with Severe Mental Illness. Issues in Mental Health Nursing 2011;32:589-97.

2.Kalkhoran S, et al.Cigarette Smoking and Quitting-Related Factors Among US Adult Health Center Patients with Serious Mental Illness. 2019:1-6.

3.Toftdahl NG, et al.Prevalence of substance use disorders in psychiatric patients: a nationwide Danish population-based study. Social Psychiatry and Psychiatric Epidemiology 2015;51:129-40.

4.Soundy A, et al.Physical activity and sedentary behaviour in outpatients with schizophrenia: a systematic review and meta-analysis. magonlinelibrarycom 2013;20:588-95. 5.Stubbs B, et al.How sedentary are people with psychosis? A systematic review and metaanalysis. Schizophrenia Research 2016;171:103-9.

6.Hahn LA, et al.Inadequate fruit and vegetable intake in people with psychosis. Australian and New Zealand Journal of Psychiatry 2014;48:1025-35.

7.De Hert M, et al.Cardiovascular disease and diabetes in people with severe mental illness: Position statement from the European Psychiatric Association. European Psychiatry 2009;24:412-24.

8.Baker AL, et al.'Better Health Choices' by telephone: A feasibility trial of improving diet and physical activity in people diagnosed with psychotic disorders. Psychiatry Research 2014;220:63-70.

9.Spring B, et al.Multiple behavior changes in diet and activity: A randomized controlled trial using mobile technology. Archives of Internal Medicine 2012;172:789-96.

10.Loughland CM, et al.Potential sampling and recruitment source impacts in schizophrenia research. Psychiatry Research 2004;125:117-27. 
11.Cabassa LJ, et al.Peer-based health interventions for people with serious mental illness: A systematic literature review. Journal of Psychiatric Research 2017;84:80-9.

12.D ELBM, et al.Peer-Delivered Recovery Support Services for Addictions in the United States: A Systematic Review. Journal of Substance Abuse Treatment 2016;63:1-9.

13.Chan A-WC, et al.Spirit 2013 statement: Defining standard protocol items for clinical trials. Annals of Internal Medicine 2013;158:200-7.

14.Eldridge SM, et al.CONSORT 2010 statement: extension to randomised pilot and feasibility trials. BMJ 2016;355.

15.Neami National. A journey shared: Annual Report 2014/15. Retrieved from http://www.neaminational.org.au/sites/default/files/201415_neami_national_annual_report_web.pdf; 2015.

16. Oades LG, et al.Leadership coaching transforming mental health systems from the inside out: The Collaborative Recovery Model as person-centred strengths based coaching psychology. International Coaching Psychology Review 2009;4:25-36.

17.Attkisson CC, et al.The client satisfaction questionnaire: Psychometric properties and correlations with service utilization and psychotherapy outcome. Evaluation and Program Planning 1982;5:233-7.

18.Lane $\mathrm{C}$, et al.Measuring adaptations of motivational interviewing: the development and validation of the behavior change counseling index (BECCI). Patient Education and Counseling 2005;56:166-73.

19.Darke S, et al. The Opiate Treatment Index (OTI) manual: Technical Report (11). Sydney, Australia: National Drug and Alcohol Research Centre; 1991.

20.Kay-Lambkin F, et al.The Impact of Tobacco Smoking on Treatment for Comorbid Depression and Alcohol Misuse. International Journal of Mental Health and Addiction 2013;11:619-33. 
PEER-DELIVERED Better Health Choices

21.Heatherton TF, et al.The Fagerstrom Test for Nicotine Dependence: a revision of the Fagerstrom Tolerance Questionnaire. The British Journal of Addiction 1991;86:1119-27. 22.Sobell LC, et al. Timeline Follow-Back. In: Litten RZ, et al., eds. Measuring Alcohol Consumption: Psychosocial and Biochemical Methods. Totowa, NJ: Humana Press; 1992:4172.

23.Collins CE, et al.The comparative validity and reproducibility of a diet quality index for adults: The Australian Recommended Food Score. Nutrients 2015;7:785-98.

24.Marshall AL, et al.Measuring total and domain-specific sitting: a study of reliability and validity. Med Sci Sports Exerc 2010;42:1094-102.

25.Craig CL, et al.International physical activity questionnaire: 12-country reliability and validity. Medicine \& Science in Sports \& Exercise 2003;35:1381-95.

26.Kroenke K, et al. The PHQ-9: Validity of a brief depression severity measure. Gen Intern Med. 2001; 16: 606-13. In.

27.Schmidt S, et al.The EUROHIS-QOL 8-item index: psychometric results of a crosscultural field study. European Journal of Public Health 2005;16:420-8.

28.Durlak J.How to select, calculate, and interpret effect sizes. Journal of pediatric psychology 2009;34:917-28.

29.Pomerleau J, et al.Interventions designed to increase adult fruit and vegetable intake can be effective: a systematic review of the literature. Nutritional Epidemiology 2005;135:248695.

30.Jansink R, et al.Minimal improvement of nurses' motivational interviewing skills in routine diabetes care one year after training: a cluster randomized trial. BMC Family Practice 2013;14:44. 
PEER-DELIVERED Better Health Choices

Table 1.

Demographic Characteristics of Participants

\begin{tabular}{|c|c|c|c|c|c|c|}
\hline \multirow[b]{2}{*}{ Variable } & \multicolumn{2}{|c|}{$\begin{array}{l}\text { Treatment } \\
(n=24)\end{array}$} & \multicolumn{2}{|c|}{$\begin{array}{l}\text { Control } \\
(n=19)\end{array}$} & \multicolumn{2}{|c|}{$\begin{array}{c}\text { Total } \\
(n=43)\end{array}$} \\
\hline & $n$ & $\%$ & $n$ & $\%$ & $n$ & $\%$ \\
\hline \multicolumn{7}{|l|}{ Gender } \\
\hline Male & 10 & 42 & 8 & 42 & 18 & 42 \\
\hline Female & 14 & 58 & 11 & 58 & 25 & 58 \\
\hline \multicolumn{7}{|l|}{ Age (y) } \\
\hline $18-35$ & 4 & 17 & 5 & 26 & 9 & 21 \\
\hline $35-50$ & 9 & 38 & 7 & 37 & 16 & 37 \\
\hline $50-65$ & 11 & 46 & 7 & 37 & 18 & 42 \\
\hline \multicolumn{7}{|l|}{ Diagnosis ${ }^{1}$} \\
\hline Psychotic disorders & 10 & 42 & 9 & 47 & 19 & 44 \\
\hline Depressive disorders & 12 & 50 & 7 & 37 & 19 & 44 \\
\hline Anxiety disorders & 11 & 46 & 6 & 32 & 17 & 40 \\
\hline $\begin{array}{l}\text { Bipolar and related } \\
\text { disorders }\end{array}$ & 4 & 17 & 4 & 21 & 8 & 19 \\
\hline $\begin{array}{l}\text { Trauma and stressor } \\
\text { related disorders }\end{array}$ & 4 & 17 & 3 & 16 & 7 & 16 \\
\hline $\begin{array}{l}\text { Obsessive-compulsive } \\
\text { and related disorders }\end{array}$ & 1 & 4 & 2 & 11 & 3 & 7 \\
\hline Personality disorders & 1 & 4 & 2 & 11 & 3 & 7 \\
\hline Other & 3 & 13 & 1 & 5 & 4 & 8 \\
\hline
\end{tabular}

Note. ${ }^{1}$ Participants were able to list more than one diagnosis. 
Total sample: outcome means at baseline, 12-weeks, and 16-weeks

\begin{tabular}{|c|c|c|c|c|c|c|}
\hline \multirow[b]{2}{*}{ Measure } & \multicolumn{3}{|c|}{ Treatment } & \multicolumn{3}{|c|}{ Control } \\
\hline & $\begin{array}{c}\text { Baseline } \\
(n=24) \\
M(S D)\end{array}$ & $\begin{array}{c}12 \text { weeks } \\
(n=13) \\
M(S D)\end{array}$ & $\begin{array}{c}16 \text { weeks } \\
(n=16) \\
M(S D)\end{array}$ & $\begin{array}{c}\text { Baseline } \\
(n=19) \\
M(S D)\end{array}$ & $\begin{array}{c}12 \text { weeks } \\
(n=14) \\
M(S D)\end{array}$ & $\begin{array}{c}16 \text { weeks } \\
(n=16) \\
M(S D)\end{array}$ \\
\hline \multicolumn{7}{|l|}{ Substance use } \\
\hline OTI tobacco use ${ }^{1^{*}}$ & $17.75(8.60)$ & $15.33(10.96)$ & $12.75(10.43)$ & $22.30(24.55)$ & $22.56(24.56)$ & $28.17(22.81)$ \\
\hline Mean cigarettes per day ${ }^{*}$ & $17.00(8.76)$ & $16.67(10.01)$ & $12.50(10.84)$ & $26.14(25.20)$ & $24.00(23.30)$ & $20.25(23.13)$ \\
\hline Standard drinks per day & $.74(2.27)$ & $.00(.00)$ & $.03(.13)$ & $.58(1.74)$ & $.86(2.21)$ & $.75(2.18)$ \\
\hline \multicolumn{7}{|l|}{ Diet } \\
\hline Fruit serves & $1.6(1.5)$ & $1.8(1.2)$ & $1.7(1.4)$ & $1.1(1.0)$ & $1.4(0.9)$ & $1.6(1.0)$ \\
\hline ARFS fruits ${ }^{2}$ & $4.7(2.4)$ & $5.0(2.0)$ & $5.6(2.6)$ & $4.1(3.5)$ & $5.6(3.0)$ & $4.6(2.6)$ \\
\hline Vegetable serves & $1.5(0.9)$ & $2.0(1.6)$ & $2.2(1.3)$ & $1.5(1.5)$ & $2.4(1.7)$ & $2.2(1.7)$ \\
\hline ARFS vegetables ${ }^{2}$ & $9.9(4.0)$ & $12.6(3.4)$ & $10.0(4.1)$ & $11.4(5.3)$ & $12.6(5.1)$ & $12.4(6.8)$ \\
\hline Leisure screen time & $283(186)$ & $229(157)$ & $218(193)$ & $247(180)$ & $272(332)$ & $310(257)$ \\
\hline \multicolumn{7}{|l|}{ Physical activity $^{3}$} \\
\hline Total Physical activity & $1081(1038)$ & $1510(1345)$ & $1638(1641)$ & 1123 (1112) & $1369(1150)$ & $1689(1780)$ \\
\hline Walking & $551(850)$ & $1016(1034)$ & $670(676)$ & $257(255)$ & 603 (798) & $688(813)$ \\
\hline Moderate activity & $210(253)$ & $453(935)$ & $603(1394)$ & $381(468)$ & $452(834)$ & 665 (1295) \\
\hline Vigorous activity & $320(644)$ & 40 (139) & $365(775)$ & $485(886)$ & $314(515)$ & $336(512)$ \\
\hline \multicolumn{7}{|l|}{ Wellbeing } \\
\hline Psychological distress $^{4}$ & $12.83(6.20)$ & $11.62(6.55)$ & $10.50(6.13)$ & $12.53(6.54)$ & $12.79(7.54)$ & $11.94(6.12)$ \\
\hline Quality of life ${ }^{5}$ & $21.96(8.23)$ & $24.92(7.09)$ & $24.69(5.72)$ & $22.53(6.74)$ & $24.93(6.86)$ & $25.47(6.78)$ \\
\hline
\end{tabular}

3 Note. ${ }^{*}$ Consistent with Baker et al ${ }^{8}$, just includes people who reported smoking in the month prior to baseline. ${ }^{1}$ Opiate Treatment Index,

$4 \quad{ }^{2}$ Australian Recommended Food Score (ARFS) index, ${ }^{3}$ International Physical Activity Questionnaire Short Form (IPAQ-SF), ${ }^{4}$ Patient Health

5 Questionnaire, ${ }^{5} \mathrm{WHO}-8$ EUROHIS Quality of Life scale. 
PEER-DELIVERED Better Health Choices

1 Table 3.

2 Total sample: Regression results for the effect of treatment on outcomes relative to the

3 control group

\begin{tabular}{|c|c|c|}
\hline & Unc & \\
\hline & Coeff (SE) & $\mathrm{p}$ \\
\hline OTI Tobacco smoking ${ }^{1}$ & & \\
\hline Treatment condition & $0.08(0.77)$ & .918 \\
\hline Time & $-0.17(0.12)$ & .141 \\
\hline Treatment condition $\mathrm{x}$ time & $-0.19(0.17)$ & .248 \\
\hline Average cigarettes per day & & \\
\hline Treatment condition & $-0.03(0.83)$ & .969 \\
\hline Time & $-0.40(0.12)$ & .001 \\
\hline Treatment condition $\mathrm{x}$ time & $-0.06(0.17)$ & .738 \\
\hline Standard drinks per day & & \\
\hline Treatment condition & $0.24(1.32)$ & .854 \\
\hline Time & $0.10(0.37)$ & .780 \\
\hline Treatment condition $\mathrm{x}$ time & $-2.02(1.64)$ & .217 \\
\hline Fruit serves & & \\
\hline Treatment condition & $-0.04(0.43)$ & .923 \\
\hline Time & $0.67(0.28)$ & .019 \\
\hline Treatment condition $\mathrm{x}$ time & $0.09(0.40)$ & .827 \\
\hline Fruit consumption $^{2}$ & & \\
\hline Treatment condition & $0.60(0.80)$ & .452 \\
\hline Time & $0.62(0.52)$ & .238 \\
\hline Treatment condition $\mathrm{x}$ time & $-0.23(0.73)$ & .750 \\
\hline Vegetable serves & & \\
\hline Treatment condition & $-0.04(0.43)$ & .923 \\
\hline Time & $0.67(0.28)$ & .019 \\
\hline Treatment condition $\mathrm{x}$ time & $0.09(0.40)$ & .827 \\
\hline Vegetable consumption ${ }^{2}$ & & \\
\hline Treatment condition & $-1.49(1.49)$ & .317 \\
\hline Time & $0.61(1.00)$ & .546 \\
\hline Treatment condition $\mathrm{x}$ time & $0.92(1.11)$ & .511 \\
\hline Leisure screen time & & \\
\hline Treatment condition & $0.13(0.27)$ & .613 \\
\hline Time & $0.18(0.02)$ & $<.001$ \\
\hline Treatment condition $\mathrm{x}$ time & $-0.40(0.03)$ & $<.001$ \\
\hline Total physical activity ${ }^{3}$ & & \\
\hline Treatment condition & $-0.04(0.37)$ & .918 \\
\hline Time & $0.40(0.01)$ & $<.001$ \\
\hline Treatment condition $\mathrm{x}$ time & $-0.03(0.01)$ & .015 \\
\hline Walking $^{3}$ & & \\
\hline Treatment condition & $0.76(0.36)$ & .037 \\
\hline Time & $0.82(0.02)$ & $<.001$ \\
\hline Treatment condition $\mathrm{x}$ time & $-0.53(0.02)$ & $<.001$ \\
\hline Moderate activity $^{3}$ & & \\
\hline Treatment condition & $-0.60(0.63)$ & .341 \\
\hline
\end{tabular}


PEER-DELIVERED Better Health Choices

Time

$0.26(0.01) \quad<.001$

Treatment condition $\mathrm{x}$ time

$0.81(0.03)$

$<.001$

Vigorous activity $^{3}$

Treatment condition

$-0.42(1.17)$

.722

Time

$0.08(0.02)$

$<.001$

Treatment condition $\mathrm{x}$ time

$-0.31(0.03)$

$<.001$

Symptom distress ${ }^{4}$

Treatment condition

$0.31(1.92)$

.873

Time

$-0.53(1.20)$

.655

Treatment condition $\mathrm{x}$ time

$-1.19(1.68)$

.477

Quality of life 5

Treatment condition

$-0.57(2.23)$

.799

Time

$2.54(1.21)$

.036

Treatment condition $\mathrm{x}$ time

$-0.71(1.70)$

.676

1 Note. SE = Standard error, ${ }^{1}$ Opiate Treatment Index, ${ }^{2}$ Australian Recommended Food Score

2 (ARFS) index, ${ }^{3}$ International Physical Activity Questionnaire Short Form (IPAQ-SF),

$3 \quad{ }^{4}$ Patient Health Questionnaire, ${ }^{5}$ WHO-8 EUROHIS Quality of Life scale. 
2 Participants who did not meet health guidelines at baseline: outcome means and effect sizes

\begin{tabular}{|c|c|c|c|c|c|c|c|c|c|c|}
\hline \multirow{2}{*}{ Measure } & \multicolumn{3}{|c|}{$\underline{\text { Treatment }}$} & \multicolumn{5}{|c|}{$\underline{\text { Control }}$} & \multirow[b]{2}{*}{$\begin{array}{l}\text { Effect } \\
\text { size }\end{array}$} & \multirow[b]{2}{*}{$95 \% \mathrm{CI}$} \\
\hline & $\begin{array}{l}\text { Baseline } \\
M(S D)\end{array}$ & $\mathrm{n}$ & $\begin{array}{l}16 \text { weeks } \\
M(S D)\end{array}$ & $\mathrm{n}$ & $\begin{array}{l}\text { Baseline } \\
M(S D)\end{array}$ & $\mathrm{n}$ & $\begin{array}{l}16 \text { weeks } \\
M(S D)\end{array}$ & $\mathrm{n}$ & & \\
\hline \multicolumn{11}{|l|}{ Substance use } \\
\hline OTI Tobacco smoking ${ }^{1}$ & $17.75(8.60)$ & 11 & $12.75(10.43)$ & 6 & $22.30(18.38)$ & 7 & $28.17(22.81)$ & 3 & $-.75^{+}$ & $-1.90, .85$ \\
\hline Standard drinks per day & $7.85(2.62)$ & 2 & - & - & $5.50(.71)$ & 2 & $6.00(2.83)$ & 2 & - & - \\
\hline \multicolumn{11}{|l|}{ Diet } \\
\hline Fruit serves & $.50(.50)$ & 13 & $1.00(.93)$ & 8 & $.46(.50)$ & 12 & $1.00(.71)$ & 9 & -.05 & $-.74, .65$ \\
\hline ARFS fruits $^{2}$ & $3.77(2.13)$ & 13 & $5.00(2.56)$ & 8 & $2.83(2.79)$ & 12 & $3.67(2.69)$ & 9 & $.15^{+}$ & $-.55, .84$ \\
\hline Vegetable serves & $1.46(.93)$ & 24 & $2.19(1.32)$ & 16 & $1.09(.94)$ & 17 & $1.71(1.20)$ & 14 & $.09^{+}$ & $-.61, .78$ \\
\hline ARFS vegetables ${ }^{2}$ & $9.88(4.03)$ & 24 & $10.00(4.08)$ & 16 & $10.29(4.43)$ & 17 & $11.50(6.81)$ & 14 & -.19 & $-.88, .51$ \\
\hline Leisure screen time & $338(167)$ & 19 & $215(198)$ & 12 & $350(120)$ & 12 & $346(191)$ & 10 & $-.61^{+}$ & $-1.31, .11$ \\
\hline \multicolumn{11}{|l|}{ Physical activity ${ }^{3}$} \\
\hline Total Physical activity & $912(984)$ & 21 & $1701(1678)$ & 15 & $687(940)$ & 13 & $1378(1818)$ & 12 & $.06^{+}$ & $-.64, .75$ \\
\hline Vigorous activity & $175(389)$ & 21 & $389(795)$ & 15 & $197(710)$ & 13 & $200(432)$ & 12 & $.33^{+}$ & $-.38,1.02$ \\
\hline Moderate activity & $200(259)$ & 21 & $643(1434)$ & 15 & $320(506)$ & 13 & $632(1460)$ & 12 & $.09^{+}$ & $-.6, .78$ \\
\hline Walking & $537(886)$ & 21 & $669(700)$ & 15 & $170(153)$ & 13 & $546(766)$ & 12 & -.33 & $-1.02, .38$ \\
\hline
\end{tabular}

3

Note. CI = confidence interval, ${ }^{+}$effect size in favour of people in the Treatment Condition. ${ }^{1}$ Opiate Treatment Index, ${ }^{2}$ Australian
Recommended Food Score (ARFS) index, ${ }^{3}$ International Physical Activity Questionnaire Short Form (IPAQ-SF). For the smoking variable, only those participants who reported smoking in the 30-days prior to the assessment were included. For the alcohol variable, only participants who reported drinking more than 2-standard drinks daily were included. For the fruit variables, only participants who reported consuming less than $2-$ servings of fruit daily were included. For the vegetable variables, only those participants who consumed less than 5-servings of vegetables daily were included. For leisure screen time, only participants who reported spending $>2$ hours leisure screen time were included. For the physical activity variables, only those participants who reported engaging in less than 5-days of moderate or vigorous activity, of at least 30-minutes in duration, were included. 
PEER-DELIVERED Better Health Choices

1

Referrals received $(\mathrm{n}=104)$

2

3

Assessed for eligibility $(\mathrm{n}=98)$

4

5
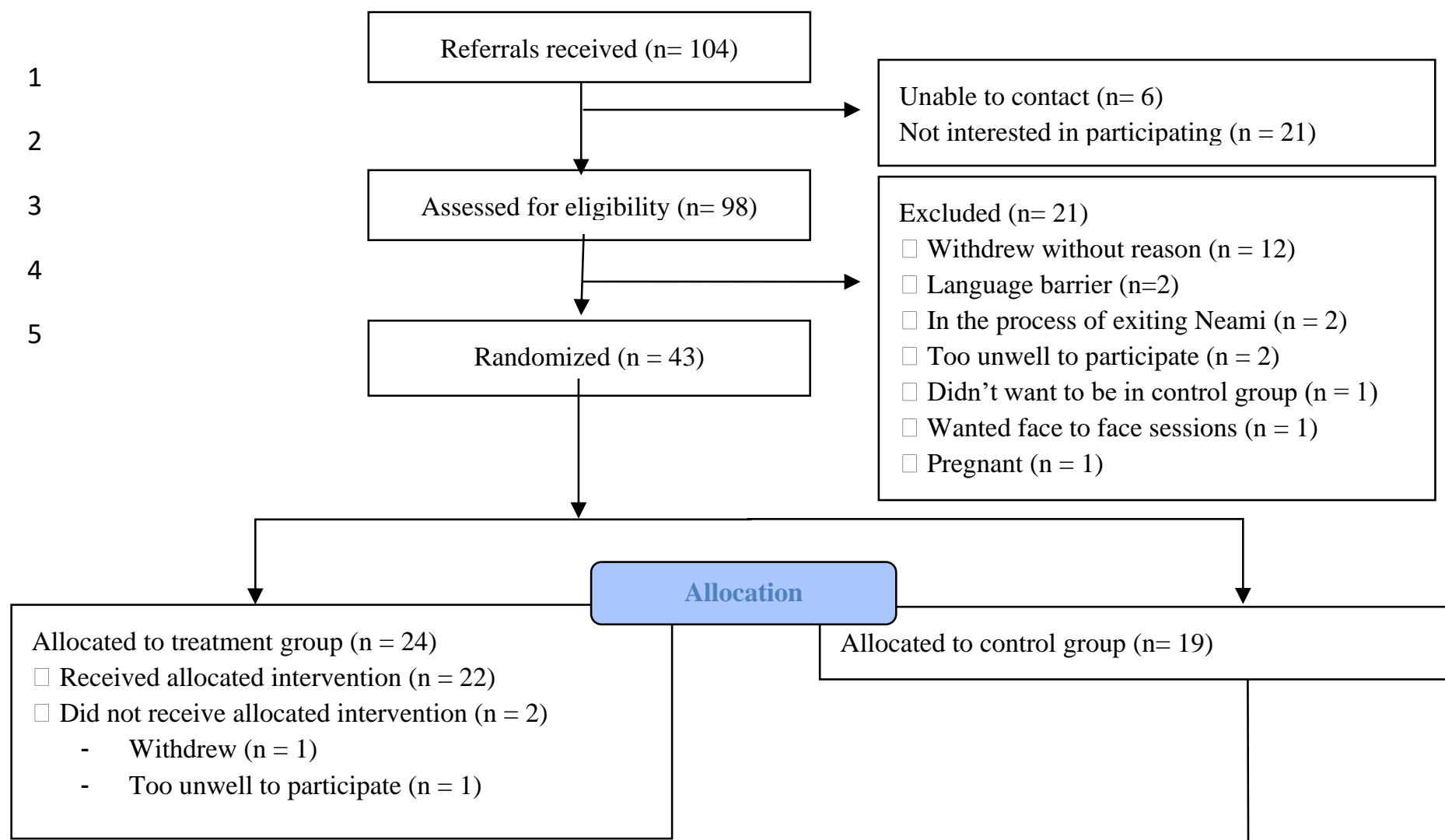

Allocation

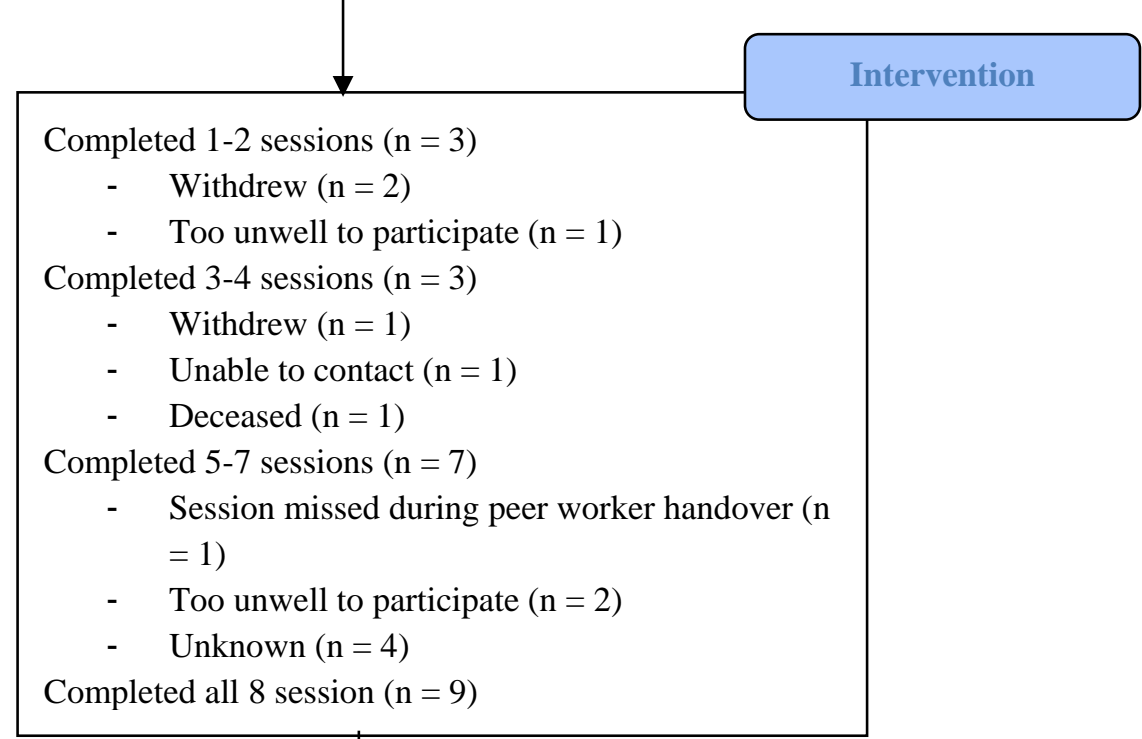

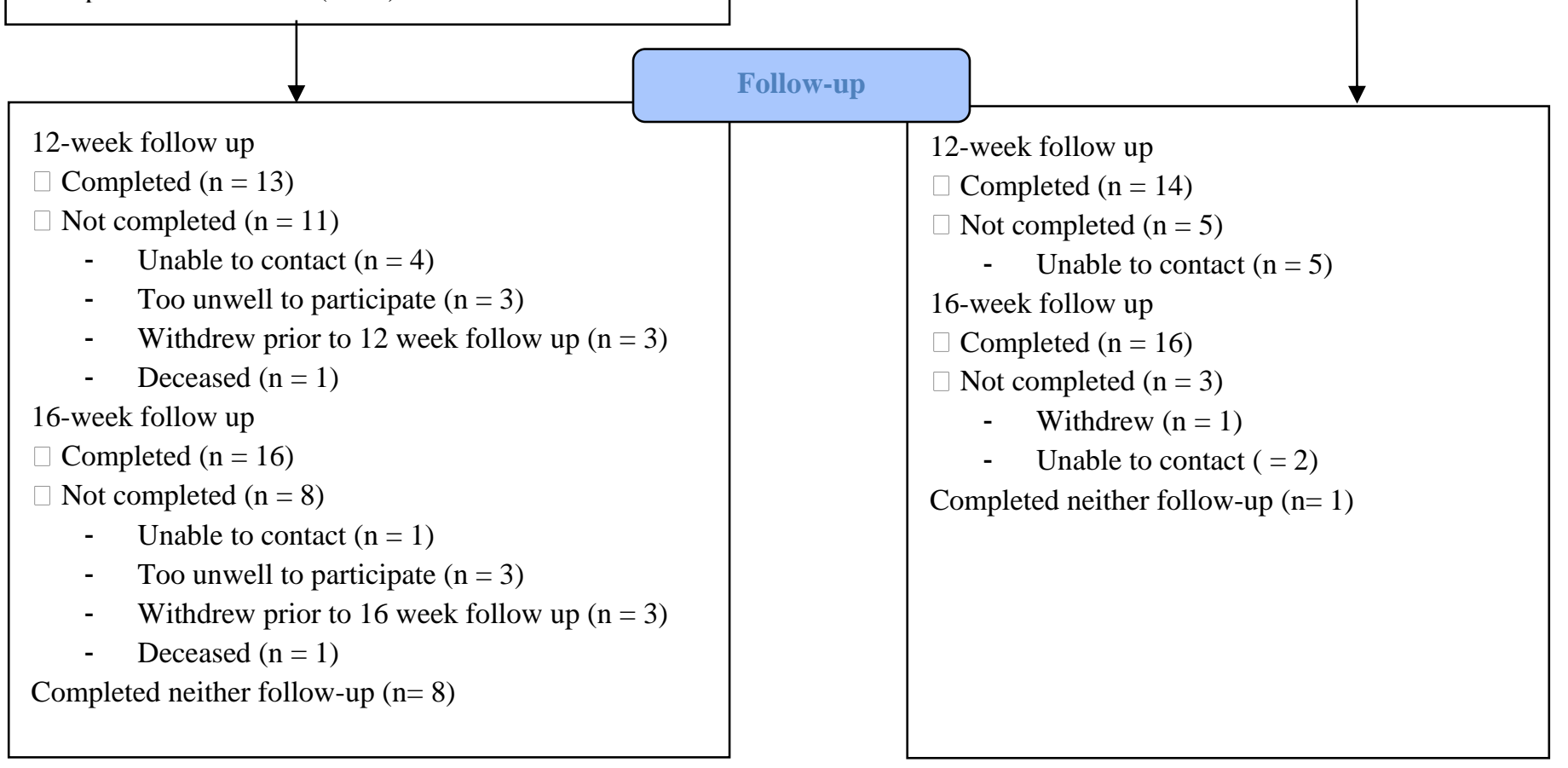

